\title{
Eficiência dos gráficos de controle xbarra, ewma e cusum
}

LiviaM ara deSouza (UFV) - liviamaradesouza@yahoo.com.br

J osél vo RibeiroJ únior (UFV)-jivo@dpi.ufv.br

Gustavo M ello Reis(UFV) - gustavo_epr@yahoo.com.br

M ayara Sayuri Ide(UFV)- mayaraide@yahoo.com.br

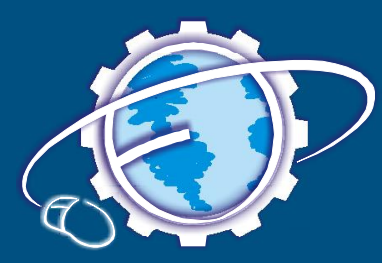

Resumo

0 planejamento dos gráficos de controle Xbarra, EWM A e CUSUM , com o objetivo de monitorar a média, deve ser feito com base nas escolhas corretas dos valores das constantes envolvidas na construção de cada gráfico, por estarem relacionados à incidência dos alarmes falso $(\alpha)$ e verdadeiro (Pd). Desse modo, visando verificar as relações de $\alpha$ e Pd em função das diferentes combinações dos valores das constantes em cada gráfico, foram simulados dados independentes de 1.000 amostras com diferentes números de repetições e sob distribuição normal com $\mu=1000$ e $\mu=100.0$ foi influenciado negativamente, de forma exponencial, pela constante $\mathrm{k}$ nos gráficos Xbarra e EWM A e, linearmente, pelas constantes $k^{*}$ e $h^{*}$, no CUSU M. Já o Pd aumenta conforme a diminuição de ke dos incrementos de n e de $\delta$, nos gráficos Xbarra eEWM A, mas não apresenta relação funcional bem definida em função das constantes $k^{*}, h^{*}$, n e $\delta$, no CU SU M . D essa forma, podem-se obter combinações dos valores das constantes capazes de gerar gráficos eficientes com $\alpha \leq 0,05$ e Pd $\geq 0,90$. E $m$ todos os três gráficos de controle é possível utilizar a estimativa de baseada nos valores de AM, R, s ou sc.

Palavras-chave: Controle estatístico, qualidade, monitoramento e variáveis.

\section{EFFICIENCY OF THE CONTROL CHARTS XBARRA, EWMA AND CUSUM}

\begin{abstract}
The planning of the control charts Xbarra, EWM A and CUSUM, aiming at mean monitoring, must be based on the correct choices of the constant values involved in the production of each chart, as they are related to the incidence of false $(\alpha)$ and true alarms $(\mathrm{Pd})$. Thus, to verify the relations of $\alpha$ and $\mathrm{Pd}$ in function of the different constant value combinations in each chart, independent data from 1,000 samples with different repetition numbers and under normal distribution with $\mu=1000$ and $\sigma=100$ were simulated ; $\alpha$ was negatively influenced, exponentially, by the constant $\mathrm{k}$ in the Xbarra and EWM A charts and linearly, by the constant $k^{*}$ and $h^{*}$, in the CUSUM chart. $O n$ the other hand, in the Xbarra and EWM A charts, $\mathrm{Pd}$ increases with $\mathrm{k}$ decrease and $\mathrm{n}$ and $\delta$ increases, without presenting a well-defined functional relationship in function of the constants $\mathrm{k}^{*}, \mathrm{~h}^{*}, \mathrm{n}$, and $\delta$ in the CUSUM. Hence, it is possible to combine the values of the constants capable of generating efficient charts, with $\alpha=0.05$ and $P d=0.90$. In all the three control charts, it is possible to utilize the $\sigma$ estimate based on the AM, R, S or sc values. Keywords: Statistical control, quality, monitoring and variables.
\end{abstract}




\section{INTRO DUÇÃO E OBJETIVO}

Atualmente, a melhoria e monitoramento da qualidade têm-se tornado uma preocupação e uma necessidade para muitas organizações (MONTGOMERY; RUNGER, 2003). Dentro do contexto de qualidade total, o controle estatístico se refere a um conjunto de ferramentas usadas com o objetivo de possibilitar a intervenção racional num sistema de produção, a fim de conferir-lhe qualidade (MUNIZ; OLIVEIRA, 2000; WERKEMA, 1995). Entre as ferramentas que compõem o controle estatístico de qualidade, os gráficos de controle são simples e eficientes no monitoramento da média e variabilidade das características de qualidade.

Os gráficos de controle mais conhecidos e utilizados são os gráficos Xbarra, EWMA (média móvel exponencialmente ponderada) e CUSUM (soma acumulativa) tabular. Existem duas medidas de eficiência associadas aos gráficos de controle, as quais são: o alarme falso ou erro alfa $(\alpha)$ e o alarme verdadeiro ou poder $(\mathrm{Pd})$. $\mathrm{O}$ alarme falso é o risco de um ponto cair fora dos limites de controle quando nenhuma causa especial estiver atuando sobre o processo, enquanto o poder é a possibilidade de um ponto cair fora dos limites de controle quando causas especiais estão atuando sobre o processo. Um gráfico de controle eficiente é aquele que possui baixos níveis de alarmes falsos e níveis altos de poder.

O gráfico mais utilizado é o gráfico Xbarra, devido à simplicidade e facilidade de construção. Os gráficos EWMA e CUSUM foram propostos com o objetivo de detectar menores diferenças, como variação especial, sem perda de eficiência. Hoje, tornou-se muito fácil a utilização de quaisquer gráficos devido às inúmeras ferramentas e softwares estatísticos disponíveis no mercado. No entanto, essa facilidade e simplicidade não vieram acompanhadas do conhecimento necessário para a sua utilização, principalmente no que se refere ao seu planejamento no qual está incluída a definição do valor das constantes de cada um dos gráficos. A grande importância da definição das constantes está no fato de elas serem capazes de influenciar medidas de eficiência (alarme falso e poder). É, portanto, fundamental conhecer a relação entre os valores das constantes com a ocorrência de alarme falso e com o poder do gráfico.

Nesse contexto, ressalta-se a importância de estimar a relação entre os valores das constantes $\mathrm{k}$ (número de desvios-padrão da média de controle que se quer detectar como variação aleatória) e n (tamanho da amos- tra) do gráfico Xbarra com as estimativas de a e Pd, o qual é proposto em função do número de desvios-padrão da média de controle que se quer detectar como variação especial $(\delta)$.

O gráfico de controle EWMA apresenta a constante $\lambda$ (peso da amostra), além das constantes k (número de desvios-padrão da média de controle que se quer detectar como variação aleatória) e n (tamanho da amostra) do gráfico de controle Xbarra de Shewhart. De acordo com Costa et al. (2004) e Montgomery (2004), o gráfico EWMA foi proposto com o objetivo de detectar menores diferenças como variação especial sem perda de eficiência. Assim, destaca-se a importância de estimar a relação entre os valores das constantes $\mathrm{k}, \lambda$ e $\mathrm{n}$ do gráfico com as estimativas dos alarmes falso e verdadeiro. Do mesmo modo, para o gráfico CUSUM tabular da média é importante estimar a relação entre os valores das constantes $\mathrm{k}^{*}$ (valor de referência padronizado), $\mathrm{h}^{*}$ (intervalo de decisão padronizado) en (tamanho da amostra) do gráfico com as estimativas dos alarmes falso e verdadeiro.

A Tabela 1 sumariza as constantes avaliadas em cada gráfico.

Tabela 1 - Constantes avaliadas por gráfico

\begin{tabular}{|l|l|}
\hline \multicolumn{1}{|c|}{ Gráfico } & \multicolumn{1}{|c|}{ Constantes } \\
\hline Xbarra & $\mathrm{k}$ e $\mathrm{n}$ \\
\hline EWMA & $\mathrm{k}, \lambda$ e $\mathrm{n}$ \\
\hline CUSUM & $\mathrm{k}^{*}, \mathrm{~h}^{*} \mathrm{e} \mathrm{n}$ \\
\hline
\end{tabular}

Outro aspecto importante do planejamento dos gráficos de controle, além da definição do valor das constantes utilizadas, é a escolha de um dos métodos para a estimativa do desvio-padrão $\sigma$, sendo AM (amplitude móvel, para $n=1$ ), $\mathrm{R}$ (amplitude, para $\mathrm{n}>1$ ), s (desvio-padrão, para $\mathrm{n}>1$ ) e sc (desvio-padrão combinado, para $\mathrm{n}>1$ ).

Os limites estimados em um estudo por gráficos de controle com o objetivo de monitorar a média são dependentes da estimativa da variabilidade aleatória do processo ou do desvio-padrão ( $\sigma)$. De acordo com Montgomery e Runger (2003), o principal objetivo do gráfico de controle é diferenciar a ocorrência de causas especiais, que provocam mudanças importantes no processo daquelas provocadas por causas comuns ou aleatórias e quantificadas pelo $\sigma$.

Entende-se que o processo está sob controle estatístico quando todos os pontos traçados no gráfico estão dentro dos dois limites de controle e se a disposição dos 
pontos é aleatória em torno da linha média (LM). Se o processo está fora de controle, um ou mais pontos se situarão fora desses limites, sob a configuração aleatória, ou haverá configurações especiais com pontos dentro ou fora dos limites de controle.

A localização de um ou mais pontos fora dos limites de controle é a indicação mais evidente da falta de controle de um processo, o qual exige intervenção em busca das causas especiais de variação. Essa regra foi proposta por Shewhart em relação aos limites de controle de $\mu_{\text {estimador }} \pm 3 \sigma_{\text {estimador }}$. No entanto, após o estabelecimento dessa regra, surgiram outras com o objetivo de aumentar a detecção de alterações. Uma delas está relacionada à flexibilidade de estabelecer valores diferentes de três para a constante $\mathrm{k}$ dos limites de controle $\left(\mu_{\text {estimador }} \pm k \sigma_{\text {estimador }}\right)$, denominada sistema $k \sigma$. As demais se referem às configurações especiais de dispersão dos pontos no gráfico de controle, que são chamadas de regras suplementares. Assim, quaisquer valores positivos de $\mathrm{k}$ até três, ou um pouco superiores ao mesmo, também podem ser usados. Nos sistemas $3 \sigma_{\text {estimador }}$, $2 \sigma_{\text {estima- }}$ dor $\mathrm{e} 1 \sigma_{\text {estimador, }}$ as probabilidades de as estimativas oriundas de determinado estimador estarem dentro dos intervalos compreendidos pelos limites inferior e superior de controle (LIC e LSC), sob causas aleatórias de variação e com base na distribuição normal, são iguais a 0,9973 ; 0,9545 ; e 0,6827 , respectivamente.

Os valores de mais ou menos $\mathrm{k}$ (valores de $\mathrm{Z}$ da distribuição normal padronizada) representam, portanto, $o$ limite de variação aleatória (processo sob controle estatístico) dos valores de um estimador de $\mathrm{Y}$ em relação à sua média $\left(\mu_{\text {estimador }}\right)$, por exemplo $\bar{Y}$, em número de desviospadrão desse estimador $\left(\sigma_{\text {estimador }}\right)$ imposto pela equipe técnica. Desse modo, a equipe técnica considera como causas aleatórias as estimativas de um estimador de Y que caem dentro do intervalo de $\mu_{\text {estimador }} \pm k \sigma_{\text {estimador }}$ e, como causas especiais, as estimativas que caem fora desse intervalo.

Um dos princípios básicos que rege a construção dos gráficos de controle estabelece que os subgrupos racionais (amostras) devem ser selecionados de modo que, à medida do possível, a variabilidade das observações dentro de uma amostra inclua toda a variabilidade aleatória e exclua a variabilidade devida a causas especiais. Nesse caso, os limites de controle $\left(\mathrm{m}_{\text {estimador }} \pm \mathrm{ks}_{\text {estimador }}\right)$ representarão limites para a variação aleatória e não para a variação especial, dado que a estimativa de $s_{\text {estimador }}$ é baseada na variação que ocorre dentro de todas as amostras. Portanto, causas especiais tenderão a gerar pontos que estejam fora dos limites de controle, enquanto a variação aleatória tender a gerar pontos que estejam dentro desses limites, pois é a variabilidade dentro da amostra que determina a largura do limite de controle. Desse modo, como cada amostra deve consistir de itens que foram produzidos tão próximos quanto possível ou dentro de um mesmo estrato, pressupõese mínima a possibilidade da presença de variabilidade devido a causas especiais e máxima a possibilidade da ocorrência de variabilidade entre as amostras, se causas especiais estiverem presentes.

Estatisticamente, pode-se verificar a eficiência de determinado gráfico de controle com base nas estimativas dos erros devidos ao alarme falso $(\alpha)$ e à falta de detecção $(\beta)$. O $\alpha$ é a probabilidade de um ponto localizado fora dos limites de controle ser devido a causas aleatórias. Ou seja, rejeita-se a hipótese nula (Ho), por exemplo, para o estimador $\bar{Y}\left(\mu^{\prime}=\mu\right)$, mas ela é verdadeira, sendo $\mu_{\mathrm{Y}}$ a média de controle. $\mathrm{O} \beta$ é a probabilidade de um ponto localizado dentro dos limites de controle ser devido a causas especiais, ou seja, aceita-se a hipótese Ho, mas ela é falsa. Portanto, o poder do gráfico $(\mathrm{Pd}=1-\beta)$ ou o alarme verdadeiro é a probabilidade de um ponto localizado fora dos limites de controle ser devido a causas especiais, ou seja, rejeita-se a hipótese Ho falsa ou aceita-se a hipótese alternativa (Ha) verdadeira. Por exemplo, para o estimador $\bar{Y}\left(\mu^{\prime}=\mu \pm \delta \sigma\right)$, sendo $\delta$ o deslocamento da média de controle $(\mu)$ que se quer detectar como variação especial, em número de desviospadrão dos valores de $\mathrm{Y}(\sigma)$ impostos pela equipe técnica, como se segue:

$$
\delta=\frac{\mu^{\prime}-\mu}{\sigma}
$$

Desse modo, o este trabalho visou estudar os gráficos de controle Xbarra de Shewhart, EWMA e CUSUM, com o objetivo de conhecer a relação entre os valores de suas constantes com a ocorrência de alarme falso, com o poder do gráfico e com a rapidez de detecção de causas especiais de variação. Além disso, será proposto um ou mais métodos de estimar, além de serem propostas normas que auxiliam a utilização correta desses gráficos em situações voltadas a diferentes interesses do monitoramento de processos nas indústrias.

\section{MATERIAL E MÉTODOS}

De forma a conhecer o relacionamento das constantes dos gráficos de controle com as estimativas de $\alpha$ e Pd, foram simulados, através do software estatístico R, 
dados independentes de 1.000 amostras com 1, 2, 4, 7, 11 e 16 repetições (n), sob a distribuição normal com $\mu=$ 1000 e $\sigma=100$. Para a utilização correta dos gráficos de controle e para a garantia de que as estimativas de $\alpha$ e Pd se manifestem, é necessário que os efeitos aleatórios da variável resposta Y sejam normais e independentemente distribuídos.

O gráfico de controle Xbarra de Shewhart é usado com base nas observações das amostras que contêm uma ou mais unidades $(\mathrm{n} \geq 1)$ de tamanhos constantes (n) ou variáveis $\left(\mathrm{n}_{\mathrm{i}}\right)$. Portanto, com base nas estimativas é possível calcular a média e o desvio-padrão de $\bar{Y}$. Para as determinações da linha média (LM) e dos limites de controle, são usadas as expressões da Tabela 2.

No sistema $k \sigma$, os parâmetros $\mu \mathrm{e}, \mathrm{ou}, \sigma_{\overline{\mathrm{Y}}}$ podem ser conhecidos ou fornecidos a priori quando for de interesse para a especificação do processo. Caso contrário, eles devem ser estimados a partir dos dados amostrais.

Após o cálculo da LM e dos limites de controle, devem-se representar os $m$ valores de $\bar{Y}_{i}$ no gráfico Xbarra e verificar se o processo pode ser considerado sob o con- trole ou se existem pontos fora dos limites e, ou, configurações não aleatórias.

Os gráficos Xbarra foram construídos com base em todas as combinações de $\mathrm{k}(1,1,5,2,2,5$ e 3$), \mathrm{n}(1,2$, $4,7,11$ e 16$)$ e $\delta(0,5,1,2$ e 3$)$. Para cada valor de $n$, foram construídos os gráficos com os valores de $\mathrm{k}$ iguais a $1,1,5,2,2,5$ e 3 .

Os valores da estatística $\mathrm{W}_{\text {i }}$ plotados no gráfico EWMA da média $(\mathrm{n} \geq 1)$ são calculados através da seguinte expressão:

$$
\mathrm{W}_{\mathrm{i}}=\lambda \overline{\mathrm{Y}}_{\mathrm{i}}+(1-\lambda) \mathrm{W}_{\mathrm{i}-1}, \text { para } \mathrm{i}=1,2, \ldots, \mathrm{m}
$$

À medida que $\lambda$ tende para 1 , esse gráfico de controle se aproxima do gráfico de controle Xbarra de Shewhart, como se segue:

$$
\mathrm{W}_{\mathrm{i}}=1 \overline{\mathrm{Y}}_{\mathrm{i}}+(1-1) \mathrm{W}_{\mathrm{i}-1}=\overline{\mathrm{Y}}_{\mathrm{i}}
$$

Caso $\mu$ seja conhecido, então tem-se $\mathrm{W}_{0}=\mu$. Caso contrário, se o valor alvo for desconhecido e substituído pelo seu estimador, então tem-se $\mathrm{W}_{0}=\overline{\mathrm{Y}}$. Para as determinações da LM e dos limites de controle, para cada amostra i são utilizadas as expressões da Tabela 3.

\begin{tabular}{|c|c|c|}
\hline & $\sigma$ conhecido & $\sigma$ desconhecido \\
\hline \multirow[t]{3}{*}{$\mu$ conhecido } & $\mathrm{LIC}=\mu-\mathrm{k} \sigma_{\overline{\mathrm{Y}}}$ & $\mathrm{LIC}=\mu-\mathrm{ks}_{\bar{Y}}$ \\
\hline & $\mathrm{LM}=\mu$ & $\mathrm{LM}=\mu$ \\
\hline & $\mathrm{LSC}=\mu+\mathrm{k} \sigma_{\bar{Y}}$ & $\mathrm{LSC}=\mu+\mathrm{kS}_{\bar{Y}}$ \\
\hline \multirow[t]{3}{*}{$\mu$ desconhecido } & $\mathrm{LIC}=\overline{\overline{\mathrm{Y}}}-\mathrm{k} \sigma_{\overline{\mathrm{Y}}}$ & $\mathrm{LIC}=\overline{\overline{\mathrm{Y}}}-\mathrm{ks}_{\overline{\mathrm{Y}}}$ \\
\hline & $\mathrm{LM}=\overline{\overline{\mathrm{Y}}}$ & $\mathrm{LM}=\overline{\overline{\mathrm{Y}}}$ \\
\hline & $\mathrm{LSC}=\overline{\overline{\mathrm{Y}}}+\mathrm{k} \sigma_{\overline{\mathrm{Y}}}$ & $\mathrm{LSC}=\overline{\overline{\mathrm{Y}}}+\mathrm{ks}_{\overline{\mathrm{Y}}}$ \\
\hline
\end{tabular}

Tabela 2 - Sistema ks para gráfico Xbarra

Tabela 3 - Sistema ks para gráfico EWMA

\begin{tabular}{|l|l|l|}
\hline & \multicolumn{1}{|c|}{$\sigma$ conhecido } & \multicolumn{1}{c|}{$\sigma$ desconhecido } \\
\hline$\mu$ conhecido & $\mathrm{LIC}_{\mathrm{i}}=\mu-\mathrm{k}_{\mathrm{Wi}}$ & $\mathrm{LIC}_{\mathrm{i}}=\mu-\mathrm{ks}_{\mathrm{Wi}}$ \\
\hline & $\mathrm{LM}=\mu$ & $\mathrm{LM}=\mu$ \\
\hline$\mu$ desconhecido & $\mathrm{LSC}_{\mathrm{i}}=\mu+\mathrm{k}_{\mathrm{Wi}}$ & $\mathrm{LSC}_{\mathrm{i}}=\mu+\mathrm{ks}_{W i}$ \\
\hline & $\mathrm{LIC}_{\mathrm{i}}=\overline{\overline{\mathrm{Y}}}-\mathrm{k}_{\mathrm{Wi}}$ & $\mathrm{LIC}_{\mathrm{i}}=\overline{\overline{\mathrm{Y}}}-\mathrm{ks}_{\mathrm{Wi}}$ \\
\hline & $\mathrm{LM}=\overline{\overline{\mathrm{Y}}}$ & $\mathrm{LM}=\overline{\overline{\mathrm{Y}}}$ \\
\hline & $\mathrm{LSC}_{\mathrm{i}}=\overline{\overline{\mathrm{Y}}}+\mathrm{k}_{\mathrm{Wi}}$ & $\mathrm{LSC}_{\mathrm{i}}=\overline{\overline{\mathrm{Y}}}+\mathrm{ks}_{\mathrm{Wi}}$ \\
\hline
\end{tabular}


No sistema $k \sigma$, os parâmetros $\mu \mathrm{e}$, ou, $\sigma_{\mathrm{Wi}}$ podem ser conhecidos ou fornecidos a priori quando for de interesse para a especificação do processo. No entanto, o conhecimento de $\sigma_{\mathrm{Wi}}$, de forma direta, é bastante complicado, dado que o pesquisador conhece a variável Y e não a variável $\mathrm{W}_{\mathrm{i}}$, para cada amostra i (Tabela 4).

Os valores de $\sigma_{\bar{Y}}$ e de $s_{\bar{Y}}$ para amostras de tamanhos variáveis e constantes são obtidos de acordo com as fórmulas apresentadas na Tabela 5.

Para $\lambda=1$, o gráfico de EWMA se reduz ao gráfico de controle Xbarra de Shewhart, e os dados anteriores à amostra avaliada deixam de ter influência no cálculo de $Y_{i}$ (COSTA et al., 2004).

Os gráficos EWMA foram construídos com base em todas as combinações de $\mathrm{k}(1 ; 1,5 ; 2 ; 2,5$; e 3$), \lambda$ $(0,0001 ; 0,25 ; 0,5 ; 0,75 ;$ e 1$), \mathrm{n}(1,2,4,7,11$, e 16$)$ e $\delta$ $(0,5 ; 1 ; 2 ;$ e 3$)$. Para cada n, construíram-se os gráficos com os seguintes valores de $\mathrm{k}(1 ; 1,5 ; 2 ; 2,5$; e 3$)$ e $\lambda$ $(0,0001 ; 0,25 ; 0,5 ; 0,75 ; \mathrm{e} 1)$.

As CUSUMs tabulares da média $(\mathrm{n} \geq 1)$, caso o valor-alvo $\mu_{\mathrm{Y}}$ seja conhecido a priori ou especificado de acordo com o interesse, são calculadas através das seguintes expressões:

$$
\begin{aligned}
& \mathrm{S}_{\mathrm{H}}(\mathrm{i})=\text { máximo }\left[0 ; \overline{\mathrm{Y}}_{\mathrm{i}}-(\mu+\mathrm{K})+\mathrm{S}_{\mathrm{H}}(\mathrm{i}-1)\right], \\
& \text { para } \mathrm{i}=1,2, \ldots, \mathrm{m} \\
& \mathrm{S}_{\mathrm{L}}(\mathrm{i})=\text { máximo }\left[0 ;(\mu-\mathrm{K})-\overline{\mathrm{Y}}_{\mathrm{i}}+\mathrm{S}_{\mathrm{L}}(\mathrm{i}-1)\right], \\
& \text { para } \mathrm{i}=1,2, \ldots, \mathrm{m}
\end{aligned}
$$

Caso o valor-alvo $\mu_{\mathrm{Y}}$ seja desconhecido, então ele deverá ser estimado a partir dos dados amostrais. Assim, as CUSUMs tabulares são calculadas através das seguintes expressões:

$$
\begin{aligned}
& \mathrm{S}_{\mathrm{H}}(\mathrm{i})=\operatorname{máximo}\left[0 ; \overline{\mathrm{Y}}_{\mathrm{i}}-(\overline{\bar{Y}}+\mathrm{K})+\mathrm{S}_{\mathrm{H}}(\mathrm{i}-1)\right], \\
& \text { para } \mathrm{i}=1,2, \ldots, \mathrm{m} \\
& \mathrm{S}_{\mathrm{L}}(\mathrm{i})=\operatorname{máximo}\left[0 ;(\overline{\bar{Y}}-\mathrm{K})-\bar{Y}_{\mathrm{i}}+\mathrm{S}_{\mathrm{L}}(\mathrm{i}-1)\right], \\
& \text { para } \mathrm{i}=1,2, \ldots, \mathrm{m}
\end{aligned}
$$

Os valores iniciais de $S_{\mathrm{H}}(0)$ e $\mathrm{SL}(0)$ são iguais a zero, e os valores de $\mathrm{S}_{\mathrm{H}}(\mathrm{i})$ e $\mathrm{S}_{\mathrm{L}}(\mathrm{i})$ acumulam desvios que são maiores do que $\mathrm{K}$, com ambas as quantidades reajustadas para zero, caso se tornarem negativos. Se pelo menos um $\mathrm{S}_{\mathrm{H}}(\mathrm{i})$ ou $\mathrm{S}_{\mathrm{L}}(\mathrm{i})$ exceder o valor de $\mathrm{H}$, então o processo será considerado como fora de controle. Desse modo, têm-se: $\mathrm{LIC}=-\mathrm{H}, \mathrm{LM}=0$ e $\mathrm{LSC}=\mathrm{H}$.

Os valores de $\mathrm{K}$ e de $\mathrm{H}$ são obtidos através das expressões apresentadas na Tabela 6 .

Tabela 4 - Desvios-padrão da variável $\mathrm{W}_{\mathrm{i}}$

\begin{tabular}{|c|c|}
\hline$\sigma$ conhecido & $\sigma$ desconhecido \\
\hline$\sigma_{\mathrm{Wi}}=\sigma_{\bar{Y}} \sqrt{\left(\frac{\lambda}{2-\lambda}\right)\left[1-(1-\lambda)^{2 \mathrm{i}}\right]}$ & $\mathrm{s}_{\mathrm{Wi}}=\mathrm{s}_{\overline{\mathrm{Y}}} \sqrt{\left(\frac{\lambda}{2-\lambda}\right)\left[1-(1-\lambda)^{2 \mathrm{i}}\right]}$ \\
\hline
\end{tabular}

Tabela 5 - Valores de $\sigma_{\bar{Y}}$ e de $\mathrm{s}_{\bar{Y}}$

\begin{tabular}{|c|c|c|}
\hline Amostras & $\sigma_{\overline{\mathrm{Y}}}$ & $\mathrm{s}_{\overline{\mathrm{Y}}}$ \\
\hline Tamanhos variáveis & $\frac{\sigma}{\sqrt{\mathrm{n}_{\mathrm{i}}}}$ & $\frac{\mathrm{s}}{\sqrt{\mathrm{n}_{\mathrm{i}}}}$ \\
\hline Tamanhos constantes & $\frac{\sigma}{\sqrt{\mathrm{n}}}$ & $\frac{\mathrm{s}}{\sqrt{\mathrm{n}}}$ \\
\hline
\end{tabular}

Tabela 6 - Valores de $\mathrm{K}$ e H

\begin{tabular}{|c|c|}
\hline$\sigma$ conhecido & $\sigma$ desconhecido \\
\hline $\mathrm{K}=\mathrm{k}^{*} \sigma_{\bar{Y}}=\mathrm{k}^{*} \frac{\sigma_{\mathrm{Y}}}{\sqrt{\mathrm{n}}}$ & $\mathrm{K}=\mathrm{k}^{*} \mathrm{~s}_{\bar{Y}}=\mathrm{k}^{*} \frac{\mathrm{s}_{\mathrm{Y}}}{\sqrt{\mathrm{n}}}$ \\
\hline $\mathrm{H}=\mathrm{h}^{*} \sigma_{\overline{\mathrm{Y}}}=\mathrm{h}^{*} \frac{\sigma_{\mathrm{Y}}}{\sqrt{\mathrm{n}}}$ & $\mathrm{H}=\mathrm{h}^{*} \mathrm{~s}_{\bar{Y}}=\mathrm{h}^{*} \frac{\mathrm{s}_{\mathrm{Y}}}{\sqrt{\mathrm{n}}}$ \\
\hline
\end{tabular}


Os gráficos CUSUM foram construídos com base em todas as combinações de $\mathrm{k}^{*}(0,25 ; 0,5 ; 1 ; \mathrm{e} 1,5), \mathrm{h}^{*}$ $(3,4,5$ e 6$), \mathrm{n}(1,2,4,7,11$ e 16$)$ e d $(0,5 ; 1 ; 2 ;$ e 3$)$. Para cada $\mathrm{n}$, construíram-se os gráficos com os seguintes valores de $\mathrm{k}^{*}(0,5 / 2,1 / 2,2 / 2$ e $3 / 2)$ e h* $(3,4,5$ e 6$)$.

As estimativas do alarme falso $(\alpha)$, em relação a todas as combinações das constantes dos gráficos, com base nas construções dos gráficos de controle com os parâmetros $\mathrm{m}$ e s, foram obtidas por meio de:

$$
\hat{\alpha}=\frac{\mathrm{NPFC}_{\delta=0}}{1000}
$$

em que:

$\mathrm{NPFC}_{\delta=0}=$ número de pontos fora de controle para $\delta=0$.

As estimativas de poder $(\mathrm{Pd})$, em relação a todas as combinações de $\mathrm{k}$, n e $\delta(0,5 ; 1 ; 2$; e 3$)$, com base nas construções dos gráficos de controle com os parâmetros $\mu^{\prime}=\mu+\delta \sigma$ e $\sigma$, foram obtidas por meio de:

$$
\hat{\mathrm{P}} \mathrm{d}=\frac{\mathrm{NPFC}_{\delta>0}}{1000}
$$

em que: $\delta>0$.

$\mathrm{NPFC}_{\delta>0}=$ número de pontos fora de controle para

A especificação dos limites de controle é uma das decisões críticas que tem de ser feita no projeto de um gráfico de controle. Pelo deslocamento dos limites de controle para mais longe da linha central, diminui-se o risco $\alpha$, ou seja, o risco de um ponto cair além dos limites de controle, indicando a condição de falta de controle quando nenhuma causa atribuída estiver presente. No entanto, o alargamento dos limites de controle aumentará também o risco $\beta$, isto é, o risco de um ponto cair entre os limites de controle, quando o processo estiver realmente fora de controle. Se se moverem os limites de controle para mais perto da linha central, o efeito oposto será obtido: o risco $\alpha$ é aumentado, enquanto o risco $\beta$ é diminuído (MONTGOMERY; RUNGER, 2003).

A consequiência de ordem prática associada ao erro $\alpha$ é intervir no processo na hora errada, quando ele está isento de causas especiais, o que em si já acarreta um custo de interrupção do processo, de mão-de-obra, além de um risco de desajustar um processo que estava ajustado. E a conseqüência de ordem prática associada ao erro $\beta$ é não intervir no processo na hora certa, quando ele está sob a influência de causas especiais (COSTA et al., 2004).

O poder do gráfico $(\mathrm{Pd}=1-\beta)$ e o alarme falso são inversamente proporcionais ao número médio de amos- tras até o sinal (NMA). Esse sinal pode ser falso ou verdadeiro. No primeiro caso, é desejável ter um NMA alto e, no segundo, um NMA baixo. O NMA é a medida de eficiência mais usual dos gráficos de controle e representa o número médio de pontos que tem de ser plotado antes de um ponto indicar uma condição fora de controle.

Após a obtenção das estimativas de $\alpha$ e Pd, foram estimadas regressões em função das constantes $\mathrm{k} e$ $\mathrm{n}$ e em função das constantes $\mathrm{k}, \mathrm{n}$ e $\delta$, respectivamente, cujos coeficientes foram testados com base no teste $\mathrm{t}$ a $5 \%$ de significância. Todos os gráficos de controle e todas as regressões foram realizados no software estatístico R.

Quanto à estimação do desvio-padrão para $\mathrm{n}=1$, foram obtidas as estimativas de $\sigma$ (sAM) por meio do método da amplitude móvel (AM) com comprimento da AM (cAM) igual a 2 para cada repetição das 10 simulações (WERKEMA, 1995; MONTGOMERY; RUNGER, 2003; COSTA et al., 2004; MONTGOMERY, 2004), dadas por:

$$
\mathrm{S}_{\mathrm{AM}}=\sum_{\mathrm{i}=2}^{1000} \frac{\mathrm{AM}_{\mathrm{i}}}{(1000-1) \mathrm{d}_{2}}=\frac{\overline{\mathrm{AM}}}{\mathrm{d}_{2}}
$$

em que:

AMi $=\mid y i-$ yi-1 $\mid=$ amplitude móvel da amostra i;

$\overline{\mathrm{AM}}=$ média da amplitude móvel;

$\mathrm{d} 2=$ constante tabelada em função do $\mathrm{cAM}=2$;

yi $=$ valor y da amostra i; e

yi-1 = valor y da amostra i- 1 .

Para os valores de $n$ iguais a 2, 4, 7, 11 e 16 , foram obtidas as estimativas de $\sigma$, por meio dos métodos da amplitude (sR), do desvio-padrão (sS) e do desvio-padrão combinado (sSC), para cada repetição das 10 simulações (WERKEMA, 1995; MONTGOMERY; RUNGER, 2003; COSTA et al., 2004; MONTGOMERY, 2004), dadas, respectivamente, por R, s e sc:

$$
\begin{aligned}
& \mathrm{s}_{\mathrm{R}}=\sum_{i=1}^{1000} \frac{\mathrm{R}_{\mathrm{i}}}{1000 \mathrm{~d}_{2}}=\frac{\overline{\mathrm{R}}}{\mathrm{d}_{2}} \\
& \mathrm{~s}_{\mathrm{S}}=\sum_{\mathrm{i}=1}^{1000} \frac{\mathrm{s}_{\mathrm{i}}}{1000 \mathrm{c}_{4}}=\frac{\overline{\mathrm{s}}}{\mathrm{c}_{4}} \\
& \mathrm{~s}_{\mathrm{SC}}=\sqrt{\frac{\sum_{\mathrm{i}=1}^{1000} \mathrm{~s}_{\mathrm{i}}^{2}}{1000}}
\end{aligned}
$$


em que:

$\mathrm{Ri}=\mathrm{y}_{\text {imaior }}-\mathrm{y}_{\text {imenor }}=$ amplitude da amostra $\mathrm{i}$;

$\overline{\mathrm{R}}=$ média da amplitude;

$s_{i}^{2}=\frac{\sum_{j=1}^{n} y_{i j}^{2}-\frac{\left(\sum_{j=1}^{n} y_{i j}\right)^{2}}{n}}{n-1}=$ variância da amostra $i$

si = desvio-padrão da amostra $\mathrm{i} ;$

$\bar{s}=$ média do desvio-padrão;

$\mathrm{y}_{\text {imaior }}=$ maior valor $\mathrm{y}$ da amostra $\mathrm{i}$;

$\mathrm{y}_{\text {imenor }}=$ menor valor $\mathrm{y}$ da amostra $\mathrm{i}$;

$\mathrm{y}_{\mathrm{ij}}=$ valor $\mathrm{x}$ da amostra i e na observação $\mathrm{j}(\mathrm{j}=1,2, \ldots, \mathrm{n})$;

$\mathrm{d}_{2}=$ constante tabelada em função do tamanho da amos-

tra $\mathrm{n}$; e

$\mathrm{c}_{4}=$ constante tabelada em função do tamanho da amostra $\mathrm{n}$.

Para o método AM com $n=1$ e para cada combinação dos métodos $\mathrm{R}$, $\mathrm{s}$ e sc com os valores de $\mathrm{n}$ iguais a $2,4,7,11$ e 16 , foram verificadas as eficiências das estimações de $s$, pelo teste $t\left(H_{0}: \mu=\sigma=100\right)$. E, para verificar o efeito de $\mathrm{n}$ nos métodos $\mathrm{R}$, $\mathrm{s}$ e sc, foram realizadas análises de regressão lineares em cada método, separadamente. As análises estatísticas foram feitas no software R a 5\% de significância.

\section{RESULTADOS E DISCUSSÃO}

Os resultados apresentados pelo gráfico Xbarra quanto aos alarmes falsos $(\alpha)$ e Poder (Pd) seguem abaixo.

$\mathrm{O} \alpha$ diminui somente em função do aumento de $\mathrm{k}$, de acordo com a seguinte equação de regressão estimada a partir do modelo exponencial (Figura 1):

$$
\hat{\alpha}=3 \times 0,11076^{\mathrm{k}}\left(\mathrm{R}^{2}=0,99\right) \text {, para } 1<\mathrm{k}<3
$$

Como se pode observar, as estimativas de diminuem, de forma mais acentuada, até aproximadamente $\mathrm{k}$ $=2$. A partir desse valor, a diminuição parece ser irrisória. Portanto, não há necessidade de ser usado $\mathrm{k}=3$ (valor mais usual) com o objetivo de minimizar o $\alpha$. Para valores de $\mathrm{k}$ iguais a $1 ; 1,5 ; 2 ; 2,5 ; \mathrm{e} 3$, as estimativas de são iguais a 0,$3323 ; 0,1106 ; 0,0368 ; 0,0122 ;$ e 0,0041 , res- pectivamente. Assim, para estimativas de $\alpha \leq 0,10$, devese ter $k \geq 1,55$; para $\alpha \leq 0,05$ deve-se ter $k \geq 1,87$; e para $\alpha \leq 0,01, k \geq 2,60$, independentemente do valor de $n$.

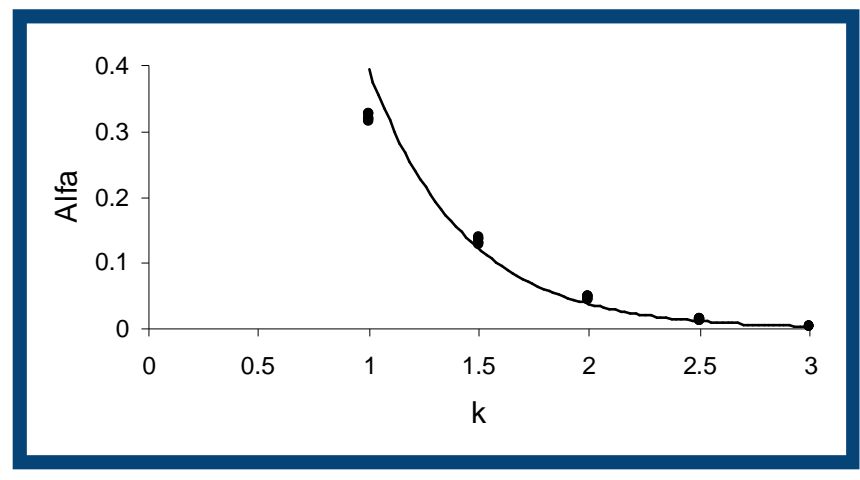

Figura 1 - Estimativas de $\alpha$ em função de k.

As estimativas de a estão bastante próximas das relatadas por King (1995), Montgomery e Runger (2003), Costa et al. (2004) e Montgomery (2004), que afirmaram também o erro a ser função apenas de k, sendo que a£0,05 e a£0,01 implicam terem-se $\mathrm{k}^{3} 1,97$ e $^{32} 2,30$, respectivamente. Do mesmo modo, elas também estão próximas das obtidas por meio das probabilidades da distribuição normal padronizada $(Z)$. Nesse último caso, para valores de a£0,10 deve-se ter $\mathrm{k}^{3} 1,65$, para a£0,05 deve-se ter $\mathrm{k}^{3} 1,96$ e para a£0,01, $\mathrm{k}^{32}, 58$, cujos valores de são obtidos por meio de:

$$
\alpha=\mathrm{P}\left(\mathrm{Z} \leq-\mathrm{k} / \mu_{\mathrm{z}}=0 \text { e } \sigma_{\mathrm{z}}=1\right)+\mathrm{P}\left(\mathrm{Z} \geq \mathrm{k} / \mu_{\mathrm{z}}=0 \text { e } \sigma_{\mathrm{z}}=1\right)
$$

$\mathrm{O}$ Pd aumenta em função da diminuição de $\mathrm{k} e$ dos aumentos de $n$ e de $\delta$, para $\delta \geq 2$ com $1 \leq n \leq 2$ e para $\delta \leq 1$, de acordo com as seguintes equações de regressão estimadas:

$$
\hat{P} d=0,2257-0,2317^{*} \mathrm{k}+0,22^{*} \mathrm{n}+0,2606^{*} \delta\left(\mathrm{R}^{2}=\right.
$$
$0,88)$, para $1 \leq \mathrm{k} \leq 3,1 \leq \mathrm{n} \leq 2$ e $2 \leq \delta \leq 3$, *Significativo pelo teste $\mathrm{t}(\mathrm{P}<0,05)$

$\hat{\mathrm{P}} \mathrm{d}=0,22125-0,25^{*} \mathrm{k}+0,0380122^{*} \mathrm{n}+0,610533^{*} \delta$ $\left(\mathrm{R}^{2}=0,89\right)$, para $1 \leq \mathrm{k} \leq 3,1 \leq \mathrm{n} \leq 16$ e $0,5 \leq \delta \leq 1$, " Significativo pelo teste $\mathrm{t}(\mathrm{P}<0,05)$

Já, para as combinações de $1 \leq \mathrm{k} \leq 3,4 \leq \mathrm{n} \leq 16$ e $2 \leq \delta \leq 3$, tem-se $\hat{P}_{d}=0,99$.

Para $P d \geq 0,90$ e $\mathrm{k} \geq 1,87(\hat{\alpha}<0,05)$, deve-se ter $\mathrm{n}$ 16 para 0,91 , enquanto para $\operatorname{Pd} 0,90$ e k $1,55(0,10)$ se deve ter $n \geq 16$ para $\delta \geq 0,76$. Nesse caso, para manter o mesmo desempenho do gráfico de controle Xbarra de Shewhart com a diminuição de $\mathrm{n}$, é necessário reduzir o valor de $\mathrm{k}$, o que pode comprometer a qualidade do gráfico no que diz respeito ao alarme falso, como já relatado por Costa et al. (2004). De acordo com Reynolds Jr. e 
Stoumbos (2004), as melhores recomendações para o gráfico de controle Xbarra de Shewhart são os valores de $n$ iguais a 4 ou 5 .

De acordo com as estimativas de $\mathrm{Pd}$, pode-se observar que para $n \geq 4$ e $\delta \geq 2$ não há influência nem do k e nem do $n$. Isso indica que nessas condições se deve estar atento apenas para as estimativas do $\alpha$. Porém, para $n=2$ só é possível obter $\operatorname{Pd} \geq 0,90$ para $\delta \geq 2$ com $\mathrm{k} \leq 1,23$ e para $\delta \geq 3$, com $\mathrm{k} \leq 2,36$. Para $\mathrm{n}=1$, só é possível obter $\mathrm{Pd} \geq 0,90$ e para $\delta \geq 3$ com $\mathrm{k} \leq 1,41$. Já nos menores valores de $\delta(\delta \leq 1)$ se devem combinar os valores de $\mathrm{k}$ e de $\mathrm{n}$ para obter boas estimativas de $\alpha$ e de Pd, simultaneamente.

Isso significa que, no início de um controle estatístico do processo (CEP), se deve dar maior atenção aos valores de $\mathrm{k}$. E, posteriormente, quando houver a necessidade de detectar como causas especiais pequenas variações na média de controle, atentar-se para os valores de $\mathrm{n}$ na presença de um k que confere baixo valor de $\alpha$.

Para estimativas de $\mathrm{Pd} \geq 0,90$ e fixando $\hat{\alpha}=0,05$ $(\mathrm{k}=1,87)$, pode-se começar o monitoramento para $\delta=3 \mathrm{com}$ $\mathrm{n}=2$, depois para $\delta=2 \mathrm{com} n=4$, independentemente do valor de k. Por fim, para $\delta=1 \mathrm{com} n=15$. Entretanto, em virtude do efeito de $\mathrm{k}$, é possível obter altas estimativas de Pd para os menores valores de $\delta$ à custa de altos valores de n. Para $\delta=0,5$, pode-se ter, no máximo, $\hat{P} d=0,88$, com $\mathrm{k} \leq 1$ e $n \geq 16$. No caso de $\delta=1$, as combinações de $k \leq 1,87$ e $\mathrm{n} \geq 15$ e de $\mathrm{k} \leq 1,55$ e $\mathrm{n} \geq 12$ possibilitam estimativas de $\operatorname{Pd} \geq 0,90$. Esses resultados vêm confirmar que o gráfico de controle Xbarra de Shewhart só deve ser indicado para detectar pequenas mudanças no processo quando se trabalhar com altos valores de n (MONTGOMERY, 2004).

A relação inversa entre $\mathrm{Pd}$ e $\mathrm{k}$ evidencia que o aumento de Pd está diretamente relacionado com o aumento de $\alpha$ para $\delta \leq 3$ e $n \leq 2$ ou para $\delta \leq 1$, independentemente do valor de n. Para estimativas de $\operatorname{Pd} \geq 0,90$ e $k=3$, o menor tamanho da amostra vale $n=4$, para $\delta \geq 2$. Já para $=1$ se pode utilizar as combinações de $k \leq 2$ e $n \geq 15, k \leq 1,5$ e $n \geq 12, k \leq 1$ e $n \geq 9$.

As recomendações gerais que incluem as estimativas de $\alpha \leq 0,05$ e de $\mathrm{Pd} \geq 0,90$ são para que se utilizem $\mathrm{k} \geq 1,87$ com os valores de $\mathrm{n} 2$ e $\delta \geq 3, \mathrm{n} \geq 4$ e $\delta \geq 2, \mathrm{n} \geq 15$ e $\delta \geq 1, n \geq 16$ e $\delta \geq 0,89$. Assim, deve-se começar um estudo por meio do gráfico de controle Xbarra de Shewhart com $\mathrm{n}=2$ ou $\mathrm{n}=4$ para detectar variações maiores como especiais $\mathrm{e}$, posteriormente, aumentar gradativamente o tamanho das amostras até $\mathrm{n}=16$, com o objetivo de detectar menores variações como especiais. Essa recomendação garante boa eficiência do gráfico e possibilita aumentar o rigor no monitoramento do processo. Caso haja interesse em detectar variações de $\delta<0,89$, deve-se fazer a escolha de outro gráfico de controle para a média do processo. Já nas estimativas de $\mathrm{Pd} \geq 0,90$ e $\mathrm{k} \leq 2,60(\hat{\alpha} \leq 0,01)$ as melhores combinações são de $n \geq 4$ e $\delta \geq 2$. Do mesmo modo, as estimativas de Pd deste trabalho estão bastante próximas daquelas obtidas por meio das probabilidades da distribuição normal padronizada. Nesse caso, se o deslocamento do $\delta$ for positivo, tem-se:

$$
\mathrm{Pd}=\mathrm{Pd}_{1}+\mathrm{Pd}_{2}
$$

em que:

$$
\begin{aligned}
& \mathrm{Pd}_{1}=\mathrm{P}\left(\mathrm{Z} \leq-\mathrm{k} / \mu_{\mathrm{z}}=\delta \sqrt{\mathrm{n}} \text { e } \sigma_{\mathrm{z}}=1\right) \\
& \mathrm{Pd}_{2}=\mathrm{P}\left(\mathrm{Z} \geq \mathrm{k} / \mu_{\mathrm{z}}=\delta \sqrt{\mathrm{n}} \text { e } \sigma_{\mathrm{z}}=1\right)
\end{aligned}
$$

De forma resumida, são apresentadas algumas estimativas de $\alpha$ e Pd do gráfico de controle Xbarra de

\begin{tabular}{|c|c|c|c|c|c|c|}
\hline \multirow[b]{3}{*}{$\mathrm{k}$} & \multirow[b]{3}{*}{$\mathrm{n}$} & \multirow[b]{3}{*}{$\hat{\alpha}$} & \multicolumn{4}{|c|}{$\hat{P d}$} \\
\hline & & & \multicolumn{4}{|c|}{$\delta$} \\
\hline & & & 0,5 & 1 & 2 & 3 \\
\hline 1,96 & 1 & 0,04 & 0,07 & 0,38 & 0,51 & 0,77 \\
\hline 1,96 & 2 & 0,04 & 0,11 & 0,42 & 0,73 & 0,99 \\
\hline 1,96 & 3 & 0,04 & 0,15 & 0,46 & 0,99 & 0,99 \\
\hline 1,96 & 4 & 0,04 & 0,19 & 0,49 & 0,99 & 0,99 \\
\hline 1,96 & 5 & 0,04 & 0,23 & 0,53 & 0,99 & 0,99 \\
\hline 1,96 & 6 & 0,04 & 0,26 & 0,57 & 0,99 & 0,99 \\
\hline 1,96 & 7 & 0,04 & 0,30 & 0,61 & 0,99 & 0,99 \\
\hline 1,96 & 8 & 0,04 & 0,34 & 0,65 & 0,99 & 0,99 \\
\hline 1,96 & 9 & 0,04 & 0,38 & 0,68 & 0,99 & 0,99 \\
\hline 1,96 & 10 & 0,04 & 0,42 & 0,72 & 0,99 & 0,99 \\
\hline 1,96 & 11 & 0,04 & 0,45 & 0,76 & 0,99 & 0,99 \\
\hline
\end{tabular}
Shewhart (Tabela 7).

Tabela 7 - Estimativas de $\alpha$ e de Pd em função de k, n e $\delta$ 
Nos gráficos de controle EWMA, as principais relações observadas entre as constantes $\mathrm{k}, \lambda$, $\mathrm{n}$ e $\delta$ e as estimativas de $\alpha$ e Pd estão relacionadas a seguir:

$\mathrm{O} \alpha$ diminui somente em função do aumento de $\mathrm{k}$, de acordo com a seguinte equação de regressão estimada a partir do modelo exponencial (Figura 2).

$$
\hat{\alpha}=2,18591 \times 0,14808^{\mathrm{k}}\left(\mathrm{R}^{2}=0,97\right) \text {, para } 1 \leq \mathrm{k} \leq 3
$$

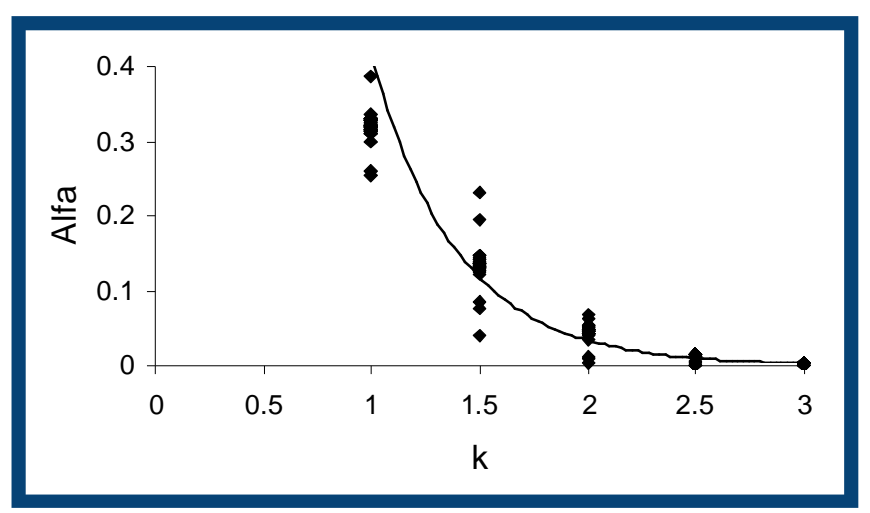

Figura 2 - Estimativas de $\alpha$ em função de k.

Como se pode observar, as estimativas de $\alpha$ diminuem, de forma mais acentuada, até aproximadamente $\mathrm{k}=2$. A partir desse valor, a diminuição parece ser irrisória. Portanto, não há necessidade de ser usado $\mathrm{k}=3$ (valor mais usual) com o objetivo de minimizar o $\alpha$. Para valores de $\mathrm{k}$ iguais a $1 ; 1,5 ; 2 ; 2,5$; e 3 , as estimativas de $\alpha$ são iguais a 0,$3237 ; 0,1246 ; 0,0479 ; 0,0184$; e 0,0071 , respectivamente. Assim, para estimativas de $\alpha \leq 0,10$ devese ter $k \geq 1,55$; para $\alpha \leq 0,05$, tr $k \geq 1,87$; e para $\alpha \leq 0,01$, $\mathrm{k} \geq 2,60$, independentemente do valor de $\mathrm{n}$.

As estimativas de $\alpha$ estão bastante próximas das relatadas por Crowder (1987), que afirmou também ser o erro $\alpha$ função apenas de k. Do mesmo modo, elas também estão próximas das obtidas por meio das probabilidades da distribuição normal padronizada (Z). Nesse último caso, para valores de $\alpha \leq 0,10$ deve-se ter $\mathrm{k} \geq 1,65$; para $\alpha \leq 0,05, \mathrm{k} \geq 1,96$; e para $\alpha \leq 0,01, \mathrm{k} \geq 2,58$, cujos valores de $\alpha$ são obtidos por meio de:

$$
\begin{aligned}
& \alpha=\mathrm{P}\left(\mathrm{Z} \leq-\mathrm{k} / \mu_{\mathrm{z}}=0 \text { e } \sigma_{\mathrm{z}}=1\right)+ \\
& +\mathrm{P}\left(\mathrm{Z} \geq \mathrm{k} / \mu_{\mathrm{z}}=0 \text { e } \sigma_{\mathrm{z}}=1\right)
\end{aligned}
$$

$\mathrm{O} \mathrm{Pd}$ aumenta em função das diminuições de $\mathrm{k} e$ de $\lambda$ e dos aumentos de $\mathrm{n}$ e de $\delta$, de acordo com as seguintes equações de regressão estimadas:

$$
\begin{aligned}
& \hat{\mathrm{P}} \mathrm{d}=0,80695-0,18335 * \mathrm{k}-0,5278 * \lambda+0,18615 * \mathrm{n}+ \\
& 0,22085 * \delta\left(\mathrm{R}^{2}=0,81\right) \text {, para } 1 \leq \mathrm{k} \leq 3,0,75 \leq \lambda \leq 1,1 \leq \mathrm{n} \leq 2 \\
& \text { e } 2 \leq \delta \leq 3,{ }^{*} \text { Significativo pelo testet }(\mathrm{P}<0,05)
\end{aligned}
$$

$\hat{\mathrm{P}} \mathrm{d}=0,737372-0,16587 * \mathrm{k}-0,56134 * \lambda+$ $0,0306199 * \mathrm{n}+0,47108 * \delta\left(\mathrm{R}^{2}=0,75\right)$, para $1 \leq \mathrm{k} \leq 3$, $0,0001 \leq \lambda \leq 1,1 \leq n \leq 16$ e $0,5 \leq \delta \leq 1$, *Significativo pelo teste $\mathrm{t}(\mathrm{P}<0,05)$

Já nas combinações de $1 \leq \mathrm{k} \leq 3, \lambda=0,0001,1 \leq \mathrm{n} \leq 16$ e $0,5 \leq \delta \leq 3$, de $1 \leq \mathrm{k} \leq 3,0,0001 \leq \lambda \leq 0,5,1 \leq \mathrm{n} \leq 2$ e $2 \leq \delta \leq 3$ e de $1 \leq \mathrm{k} \leq 3,0,75 \leq \lambda \leq 1,4 \leq \mathrm{n} \leq 16$ e $2 \leq \delta \leq 3$, tem-se $\hat{\mathrm{P}} \mathrm{d}=0,99$.

Para $\operatorname{Pd} \geq 0,90$ com $\mathrm{k} \geq 1,87$ ( $\hat{\alpha} \leq 0,05)$, deve-se ter $0,75 \leq \lambda \leq 0,78$ e $n \geq 1$ para $\geq 3$; ou $\lambda \leq 0,0011$ e $n \geq 1$ para $\delta \geq 0,94$; ou $\lambda \leq 0,0053$ e $n \geq 2$ para $\delta \geq 0,88$; ou $\lambda=0,0001$ e $\mathrm{n} \geq 1$ para $\delta \geq 0,5$; ou $\lambda \leq 0,5$ e $\mathrm{n} \geq 1$ para $\delta \geq 2$; ou $\lambda \geq 0,75$ e $\mathrm{n} \geq 4$ para $\delta \geq 2$. Já para $\mathrm{Pd} \geq 0,90$ e $\mathrm{k} \geq 2,60(\hat{\alpha} \leq 0,01)$ se devem ter $0,75 \leq \lambda \leq 0,90$ e $n \geq 2$ para $\delta \geq 3$; ou $\lambda \leq 0,0078$ e $\mathrm{n} \geq 2$ para $\delta \geq 1,14$; ou $\lambda=0,0001$ e $\mathrm{n} \geq 1$ para $\delta \geq 0,5$; ou $\lambda \leq 0,5$ e $n \geq 1$ para $\delta \geq 2$; ou $\lambda \geq 0,75$ e $n \geq 4$ para $\delta \geq 2$. Nesse caso, para manter o mesmo desempenho do gráfico de controle EWMA com a diminuição de n, é necessário reduzir o valor de $\lambda$, sem que haja o comprometimento da qualidade do gráfico no que diz respeito ao alarme falso.

Como se pode observar, é possível obter estimativas de $\mathrm{Pd} \geq 0,90$ e $\alpha \leq 0,05(\mathrm{k} \geq 1,87)$ para $\mathrm{n}=1$, nas seguintes condições: $0,75 \leq \lambda \leq 0,78$ e $\delta \geq 3, \lambda \leq 0,0011$ e $\delta \geq 0,94, \lambda=0,0001$ e $\delta \geq 0,5, \lambda \leq 0,5$ e $\delta \geq 2$. Isso significa que, no início ou na etapa final de um controle estatístico do processo (CEP), se deve dar maior atenção aos valores de $\lambda$. Portanto, quando houver a necessidade de detectar, como causas especiais, grandes variações na média de controle, deve-se utilizar $\lambda \geq 0,5$, caso contrário, quando houver a necessidade de detectar pequenas variações com $\lambda \leq 0,0011$. Esses resultados vêm confirmar que o gráfico de controle EWMA pode ser indicado para detectar grandes ou pequenas mudanças no processo, mesmo para $\mathrm{n}=1$. Portanto, a correta utilização da constante $\lambda$ no gráfico EWMA torna possível obter altas estimativas de Pd para $n=1$ e $\alpha \leq 0,05$.

De forma resumida, são apresentadas algumas estimativas de $\alpha$ e Pd, do gráfico de controle EWMA (Tabela 8).

No gráfico CUSUM, o $\alpha$ diminui em função dos aumentos de $\mathrm{k}^{*} \mathrm{e} \mathrm{h}^{*}$, mas não sofre efeito do $\mathrm{n}$, de acordo com a seguinte equação de regressão linear estimada:

$$
\begin{aligned}
& \hat{\alpha}=0,59675-0,665667 * \mathrm{k}^{*}-0,05425 * \mathrm{~h}^{*}\left(\mathrm{R}^{2}=\right. \\
& 0,73) \text {, para } 0,25 \leq \mathrm{k} * 0,5 \mathrm{e} 3 \mathrm{~h} * 6,{ }^{*} \text { Significativo pelo } \\
& \text { teste } \mathrm{t}(\mathrm{P}<0,05) \\
& \text { Já nas combinações de } 1 \leq \mathrm{k} * \leq 1,5 \text { e } 3 \leq \mathrm{h} * \leq 6 \text { tem- }
\end{aligned}
$$
$\mathrm{se}=\hat{\alpha} 0,0002$. 
Tabela 8 - Estimativas de a e de Pd em função de k, 1, n e d

\begin{tabular}{|c|c|c|c|c|c|c|c|}
\hline \multirow[b]{3}{*}{$\mathrm{k}$} & \multirow[b]{3}{*}{$\lambda$} & \multirow[b]{3}{*}{$\mathrm{n}$} & \multirow[b]{3}{*}{$\hat{\alpha}$} & \multicolumn{4}{|c|}{$\hat{P d}$} \\
\hline & & & & \multicolumn{4}{|c|}{$\delta$} \\
\hline & & & & 0,5 & 1 & 2 & 3 \\
\hline 1,96 & 0,0001 & 1 & 0,05 & 0,99 & 0,99 & 0,99 & 0,99 \\
\hline 1,96 & 0,0001 & 2 & 0,05 & 0,99 & 0,99 & 0,99 & 0,99 \\
\hline 1,96 & 0,0001 & 3 & 0,05 & 0,99 & 0,99 & 0,99 & 0,99 \\
\hline 1,96 & 0,0001 & 4 & 0,05 & 0,99 & 0,99 & 0,99 & 0,99 \\
\hline 1,96 & 0,25 & 1 & 0,05 & 0,54 & 0,77 & 1 & 1 \\
\hline 1,96 & 0,25 & 2 & 0,05 & 0,57 & 0,80 & 1 & 1 \\
\hline 1,96 & 0,25 & 3 & 0,05 & 0,60 & 0,83 & 1 & 1 \\
\hline 1,96 & 0,25 & 4 & 0,05 & 0,63 & 0,87 & 1 & 1 \\
\hline 1,96 & 0,5 & 1 & 0,05 & 0,40 & 0,63 & 0,99 & 0,99 \\
\hline 1,96 & 0,5 & 2 & 0,05 & 0,43 & 0,66 & 0,99 & 0,99 \\
\hline 1,96 & 0,5 & 3 & 0,05 & 0,46 & 0,69 & - & - \\
\hline 1,96 & 0,5 & 4 & 0,05 & 0,49 & 0,73 & 0,99 & 0,99 \\
\hline 1,96 & 0,75 & 1 & 0,05 & 0,26 & 0,49 & 0,68 & 0,90 \\
\hline 1,96 & 0,75 & 2 & 0,05 & 0,29 & 0,52 & 0,87 & 1 \\
\hline 1,96 & 0,75 & 3 & 0,05 & 0,32 & 0,55 & - & - \\
\hline 1,96 & 0,75 & 4 & 0,05 & 0,35 & 0,58 & 0,99 & 0,99 \\
\hline 1,96 & 1 & 1 & 0,05 & 0,12 & 0,35 & 0,55 & 0,77 \\
\hline 1,96 & 1 & 2 & 0,05 & 0,15 & 0,38 & 0,73 & 0,95 \\
\hline 1,96 & 1 & 3 & 0,05 & 0,18 & 0,41 & - & - \\
\hline 1,96 & 1 & 4 & 0,05 & 0,21 & 0,44 & 0,99 & 0,99 \\
\hline
\end{tabular}

De acordo com a equação de regressão, as estimativas de $\alpha$ são mais influenciadas pelo $\mathrm{k}^{*}$. Isso implica o fato de que, se houver o objetivo de minimizálo, se deve dar maior atenção a essa constante. Note-se que para $\mathrm{k}^{*} \geq 1$, independentemente dos valores de $\mathrm{h}^{*} \mathrm{e}$ $\mathrm{n}$, tem-se $\bar{\alpha}=0,0002$. Para $\mathrm{k}^{*}=0,5$, as estimativas de $\alpha$ são iguais a $0,1012\left(h^{*}=3\right), 0,0469\left(h^{*}=4\right)$ e $0(5 \leq h * \leq 6)$. Já para $\mathrm{k}^{*}=0,25$ as estimativas de $\alpha$ são iguais a 0,2676 $\left(h^{*}=3\right), 0,2133\left(h^{*}=4\right), 0,1591 \quad\left(h^{*}=5\right)$ e $0,1048\left(h^{*}=6\right)$, independentemente do valor de $n$.

As estimativas deste trabalho são semelhantes às obtidas por Hawkins (1992) e King (1995), que mostraram, também, que as alterações em $\mathrm{k}^{*}$ são mais importantes que as de $h^{*}$, no sentido de promoverem mudanças no alarme falso.

Já o Pd não apresenta relação funcional bem definida em função das constantes $\mathrm{k}^{*}, \mathrm{~h}^{*}$, $\mathrm{n}$ e $\delta$. Desse modo, foram obtidas as seguintes estimativas de $\mathrm{Pd}$ :

$\hat{\mathrm{P}} \mathrm{d}=0,97$, para $0,25 \leq \mathrm{k}^{*} \leq 1,5,3 \leq \mathrm{h}^{*} \leq 6$,

$1 \leq \mathrm{n} \leq 16$ e $1 \leq \delta \leq 3$

$\hat{\mathrm{P}} \mathrm{d}=0,94$, para $0,25 \leq \mathrm{k}^{*} \leq 0,5,3 \leq \mathrm{h}^{*} \leq 6$,

$1 \leq \mathrm{n} \leq 16$ e $\delta=0,5$

$$
\begin{aligned}
& \hat{\mathrm{P}} \mathrm{d}=0,99, \text { para } \mathrm{k}^{*}=1,3 \leq \mathrm{h}^{*} \leq 6,7 \leq \mathrm{n} \leq 16 \\
& \text { e } \delta=0,5 \\
& \hat{\mathrm{P}} \mathrm{d}=0,66, \text { para }^{*}=1,3 \leq \mathrm{h}^{*} \leq 6, \mathrm{n}=4 \mathrm{e} \\
& \delta=0,5 \\
& \hat{\mathrm{P}} \mathrm{d}=0,01, \text { para } \mathrm{k}^{*}=1,3 \leq \mathrm{h}^{*} \leq 6,1 \leq \mathrm{n} \leq 2 \mathrm{e} \\
& \delta=0,5 \\
& \hat{\mathrm{P}} \mathrm{d}=0,97, \text { para } \mathrm{k}^{*}=1,5,3 \leq \mathrm{h}^{*} \leq 6,11 \leq \mathrm{n} \leq 16 \mathrm{e} \\
& \delta=0,5 \\
& \hat{\mathrm{P}} \mathrm{d}=0,14, \text { para } \mathrm{k}^{*}=1,5,3 \leq \mathrm{h}^{*} \leq 6, \mathrm{n}=7 \mathrm{e} \\
& \delta=0,5 \\
& \hat{\mathrm{P}} \mathrm{d}=0,004, \text { para } \mathrm{k}^{*}=1,5,3 \leq \mathrm{h}^{*} \leq 6,1 \leq \mathrm{n} \leq 4 \text { e } \\
& \delta=0,5
\end{aligned}
$$

Portanto, para $\delta \geq 1$ o Pd é próximo de 1 , independentemente das combinações de $\mathrm{k}^{*}, \mathrm{~h}^{*}$ e $\mathrm{n}, \mathrm{o}$ que não possibilita discriminar mudanças especiais na média de controle acima desse deslocamento, como para $\delta \geq 2$ ou $\delta \geq 3$. Desse modo, caso haja o interesse em discriminá-los nas primeiras etapas do CEP, deve-se fazer uso do gráfico de controle Xbarra de Shewhart. Entretanto, o gráfico de controle CUSUM tabular da média permite detectar, com eficiência, pequenos 
deslocamentos especiais da média $(<1)$, em função das baixas e altas estimativas de $\alpha$ e $\mathrm{Pd}$, respectivamente, mesmo para $n=1$. Para estimativas de $\operatorname{Pd} \geq 0,90$ e $\alpha \leq 0,05$, podem-se utilizar $\mathrm{k}^{*} \geq 0,58, \mathrm{~h} * \geq 3$ e $\mathrm{n} \geq 1$, para $\delta \geq 1$. Já para $\delta \geq 0,5$, podem-se utilizar $\mathrm{k}^{*}=0,5, \mathrm{~h}^{*} \geq 4$ e $\mathrm{n} \geq 1$ ou $\mathrm{k}^{*} \geq 0,34$, $h^{*}=6$ e $n \geq 1$. Para estimativas de $\operatorname{Pd} \geq 0,90$ e $\alpha \leq 0,01$, podem-se utilizar $\mathrm{k}^{*} \geq 1, \mathrm{~h}^{*} \geq 3$ e $\mathrm{n} \geq 1$, para $\delta \geq 1$. Já para $\delta \geq 0,5$ se podem utilizar $k^{*}=1, h^{*} \geq 3$ e $n \geq 7$ ou k* $* 1,5, h^{*} \geq 3$ e $n \geq 11$. Portanto, deve-se começar um estudo por meio desse gráfico de controle com $\mathrm{k}^{*}=1, \mathrm{~h}^{*}=6 \mathrm{e} \mathrm{n}=1 \mathrm{e}$, posteriormente, diminuir gradativamente o valor de $\mathrm{k}^{*}$ até 0,34 , com o objetivo de detectar, cada vez mais, menores variações como especiais. Assim, o gráfico CUSUM deve ser implementado nas etapas finais do CEP, quando pode ser interessante detectar menores diferenças como especiais.

De forma resumida, são apresentadas algumas estimativas de $\alpha$ e Pd, do gráfico de controle CUSUM tabular (Tabela 9).

De acordo com Reynolds Jr. e Stoumbos (2004), o gráfico CUSUM tabular da média tem bom desempenho em detectar pequenos ou grandes deslocamentos na média de controle, mesmo para $n=1$. Eles consideraram que esse gráfico tem melhor desempenho do que o Xbarra de Shewhart, sendo similar ao gráfico de controle da média móvel ponderada exponencialmente (EWMA).
Assim, um gráfico de controle bem planejado é aquele que apresenta, teoricamente, baixo alarme falso $(\alpha \leq 0,10$ ou $\alpha \leq 0,05$ ou $\alpha \leq 0,01)$, alto poder $(\operatorname{Pd} \geq 0,90$ ou $\operatorname{Pd} \geq 0,95$ ou $\mathrm{Pd} \geq 0,99$ ), alto $\mathrm{NMA}_{\delta=0}$ e baixo $\mathrm{NMA}_{\delta>0}$, em função do $\delta$ preestabelecido pela equipe técnica. Nesse caso, recomendam-se escolher valores de $\delta \geq 3$, no início do controle de qualidade. Para $2<\delta<3$ e $1 \leq \delta \leq 2$, nas fases intermediárias e, para $\delta \leq 1$, quando o controle de qualidade já se encontra numa fase mais adiantada, no tocante à redução da variabilidade do processo. Assim, no início, são controladas grandes variações devidas às causas especiais e, com o passar do tempo, buscam-se detectar efeitos cada vez menores dessas possíveis causas, se houver necessidade.

Em relação à definição de um método (AM, R, $\mathrm{s}$ ou sc) para a estimativa do desvio-padrão $\sigma$, para $n=1$ e na ausência de causas especiais, ou seja, quando o processo está sob o controle estatístico em relação à média, o método de $\mathrm{AM}$ forneceu uma estimativa que não diferiu $(\mathrm{P}>0,05)$ de $\sigma(\mathrm{sAM}=100,10)$. No entanto, na prática, como não se sabe a priori se haverá ou não causas especiais interferindo no processo, é importante estar atento aos limites de controle estimados no gráfico AM. Uma alternativa de diminuir os efeitos das causas especiais na estimativa de $\sigma$ é estimá-los com base nos valores das amostras ordenados de forma crescente.

Tabela 9 - Estimativas de a e Pd em função de k*, h*, n e d

\begin{tabular}{|c|c|c|c|c|c|c|c|}
\hline & & & & \multicolumn{4}{|c|}{$\hat{P} d$} \\
\hline & & & & \multicolumn{4}{|c|}{$\delta$} \\
\hline $\mathrm{k}^{*}$ & $\mathrm{~h}^{*}$ & $\mathrm{n}$ & $\hat{\alpha}$ & 0,5 & 1 & 2 & 3 \\
\hline 0,25 & 6 & 1 & 0,10 & 0,94 & 0,97 & 0,97 & 0,97 \\
\hline 0,25 & 6 & 2 & 0,10 & 0,94 & 0,97 & 0,97 & 0,97 \\
\hline 0,25 & 6 & 3 & 0,10 & 0,94 & 0,97 & 0,97 & 0,97 \\
\hline 0,25 & 6 & 4 & 0,10 & 0,94 & 0,97 & 0,97 & 0,97 \\
\hline 0,5 & 6 & 1 & 0 & 0,94 & 0,97 & 0,97 & 0,97 \\
\hline 0,5 & 6 & 2 & 0 & 0,94 & 0,97 & 0,97 & 0,97 \\
\hline 0,5 & 6 & 3 & 0 & 0,94 & 0,97 & 0,97 & 0,97 \\
\hline 0,5 & 6 & 4 & 0 & 0,94 & 0,97 & 0,97 & 0,97 \\
\hline 1 & 6 & 1 & 0,0002 & 0,01 & 0,97 & 0,97 & 0,97 \\
\hline 1 & 6 & 2 & 0,0002 & 0,01 & 0,97 & 0,97 & 0,97 \\
\hline 1 & 6 & 3 & 0,0002 & - & 0,97 & 0,97 & 0,97 \\
\hline 1 & 6 & 4 & 0,0002 & 0,66 & 0,97 & 0,97 & 0,97 \\
\hline 1,5 & 6 & 1 & 0,0002 & 0,004 & 0,97 & 0,97 & 0,97 \\
\hline 1,5 & 6 & 2 & 0,0002 & 0,004 & 0,97 & 0,97 & 0,97 \\
\hline 1,5 & 6 & 3 & 0,0002 & 0,004 & 0,97 & 0,97 & 0,97 \\
\hline 1,5 & 6 & 4 & 0,0002 & 0,004 & 0,97 & 0,97 & 0,97 \\
\hline
\end{tabular}


Para $\mathrm{n} \geq 2$, os métodos $\mathrm{R}(\mathrm{sR}=99,68), \mathrm{s}(\mathrm{sS}=100,09)$ e sc $(\mathrm{sSC}=99,58)$ forneceram estimativas que não diferiram $(\mathrm{P}>0,05)$ de $\sigma=100$. Além disso, não houve efeito $(\mathrm{P}>0,05)$ de $\mathrm{n}$ sobre elas.

Como mencionado anteriormente, os gráficos de controle são ferramentas simples de serem construídas e utilizadas. Porém, essa simplicidade acarretou em uso indiscriminado desses gráficos, muitas vezes sem um bom conhecimento dos conceitos estatísticos em que eles se baseiam. Dessa forma, considera-se de fundamental importância o estudo dos tipos de gráficos de controle e também o conhecimento necessário para a utilização dos mesmos. A falta de informação sobre as formas certas de uso e construção dos gráficos pode acarretar em uma intervenção desnecessária sobre o processo, trazendo, com isso, prejuízos e perdas de tempo para as empresas. Portanto, com base nos resultados deste trabalho, é possível caracterizar a aplicação dos gráficos de controle Xbarra de Shewhart, EWMA e CUSUM, em função de diferentes interesses sobre o monitoramento de processos. Além disso, é possível determinar as vantagens e as desvantagens dos diferentes gráficos de controle e estabelecer valores das constantes para a construção desses gráficos que promovam condições mais satisfatórias em relação aos diferentes interesses de monitoramento.

Em resumo, é importante identificar os melhores valores das constantes de construção dos gráficos de controle e avaliar o efeito dessas escolhas sobre a ocorrência de alarme falso e da falta de detecção de descontrole nos sistemas produtivos, de acordo com os interesses da equipe técnica da empresa.

\section{CONCLUSÕES}

No planejamento de um gráfico de controle Xbarra de Shewhart, algumas orientações devem ser seguidas para se obter um gráfico que confere baixas e altas estimativas de $\alpha$ e Pd, respectivamente. Com base nas estimativas de $\alpha \leq 0,05$, pode-se utilizar, no mínimo, $\mathrm{k}=$ 1,87. Nesse caso, para estimativas de $\mathrm{Pd} \geq 0,90 \mathrm{com}$ $\mathrm{k} \geq 1,87$, as melhores combinações são de $\mathrm{n} \geq 2$ e $\delta \geq 3, \mathrm{n} \geq 4$ e $\delta \geq 2, n \geq 15$ e $\delta \geq 1, n \geq 16$ e $\delta \geq 0,89$ (Tabela 10 ).

Desse modo, o gráfico de controle Xbarra só é eficiente para detectar pequenas variações especiais à custa de um n relativamente alto. No planejamento de um gráfico de controle EWMA, recomendam-se as seguintes orientações serem seguidas para a obtenção um gráfico com baixas e altas estimativas de $\alpha$ e Pd, respectivamente. Com base nas estimativas de $\alpha 0,05$, pode-se utilizar, no mínimo, $\mathrm{k}=1,87$. Nesse caso, para estimativas de $\operatorname{Pd} \geq 0,90$ com $\mathrm{k} \geq 1,87$, as melhores combinações são de $0,75 \leq \lambda \leq 0,78$ e $n \geq 1$ para $\delta \geq 3$ ou $\lambda \leq 0,0011$ e $n \geq 1$ para $\delta \geq 0,94$ ou $\lambda \leq 0,0053$ e $n \geq 2$ para $\delta \geq 0,88$ ou $\lambda=0,0001$ e $n \geq 1$ para $\delta \geq 0,5$ ou $\lambda \leq 0,5$ e $n \geq 1$ para $\delta \geq 2$ ou $\lambda \geq 0,75$ e $n \geq 4$ para $\delta \geq 2$ (Tabela 11).

Tabela 10 - Resumo das constantes $\mathrm{k}, \mathrm{n}$ e $\delta \alpha \leq 0,05$ e $\mathrm{Pd} \geq 0,90$ no gráfico Xbarra de Shewhart

\begin{tabular}{|c|c|c|}
\hline \multicolumn{3}{|c|}{$\alpha \leq 0,05$ e $\mathrm{Pd} \geq 0,90$} \\
\hline $\mathrm{k}$ & $\mathrm{n}$ & $\delta$ \\
\hline$\geq 1,87$ & $\geq 2$ & $\geq 3$ \\
\hline$\geq 1,87$ & $\geq 4$ & $\geq 2$ \\
\hline$\geq 1,87$ & $\geq 15$ & $\geq 1$ \\
\hline$\geq 1,87$ & $\geq 16$ & $\geq 0,89$ \\
\hline
\end{tabular}

Tabela 11 - Resumo das constantes $\mathrm{k}, \lambda$, n e $\delta$ para $\alpha 0,05$ e $\operatorname{Pd} \geq 0,90$ no gráfico EWMA

\begin{tabular}{|c|c|c|c|}
\hline \multicolumn{4}{|c|}{$\alpha \leq 0,05$ e $\mathrm{Pd} \geq 0,90$} \\
\hline $\mathrm{k}$ & $\lambda$ & $\mathrm{n}$ & $\delta$ \\
\hline$\geq 1,87$ & $\geq 0,75$ e $\leq 0,78$ & $\geq 1$ & $\geq 3$ \\
\hline$\geq 1,87$ & $\leq 0,0011$ & $\geq 1$ & $\geq 0,94$ \\
\hline$\geq 1,87$ & $\leq 0,0053$ & $\geq 2$ & $\geq 0,88$ \\
\hline$\geq 1,87$ & 0,0001 & $\geq 1$ & $\geq 0,5$ \\
\hline$\geq 1,87$ & $\leq 0,5$ & $\geq 1$ & $\geq 2$ \\
\hline$\geq 1,87$ & $\geq 0,75$ & $\geq 4$ & $\geq 2$ \\
\hline
\end{tabular}

Desse modo, o gráfico de controle EWMA é eficiente para detectar pequenas ou grandes variações especiais, mesmo para $n=1$. Por fim, no planejamento de um gráfico de controle CUSUM tabular, as orientações a serem seguidas para se obter um gráfico que confere baixas e altas estimativas de $\alpha$ e Pd, respectivamente, são: para estimativas de $\operatorname{Pd} \geq 0,90$ e $\alpha \leq 0,05$, podem-se utilizar $\mathrm{k}^{*} \geq 0,58, \mathrm{~h}^{*} \geq 3$ e $\mathrm{n} \geq 1$, para $\delta \geq 1$. Já, para $\delta=0,5$, podem-se utilizar $\mathrm{k}^{*}=0,5, \mathrm{~h}^{*} \geq 4 \mathrm{e} \mathrm{n} \geq 1$ ou $\mathrm{k}^{*} \geq 0,34, \mathrm{~h}^{*}=6$ e $n \geq 1$ (Tabela 12).

Desse modo, o gráfico de controle CUSUM tabular é eficiente para detectar pequenas ou grandes variações especiais $(\delta \geq 0,5)$ na média de controle, mesmo para $\mathrm{n}=$ 1. Para um planejamento de um gráfico de controle, as orientações para a definição de um método para a estimativa de desvio-padrão são: para $\mathrm{n}=1$, a estimativa do desvio-padrão pela AM se aproxima do verdadeiro 
parâmetro $\sigma$, na ausência de causas especiais e, para $\mathrm{n} \geq 2$, ela pode ser obtida com base nos métodos $\mathrm{R}, \mathrm{s}$ ou sc. Nesse último caso, a escolha pode ser baseada em critérios pessoais.

Tabela 12 - Resumo das constantes $\mathrm{k}^{*}, \mathrm{~h} *$, n e $\delta$ para $\alpha \leq 0,05$ e $\mathrm{Pd} \geq 0,90$ no gráfico CUSUM

\begin{tabular}{|c|c|c|c|}
\hline \multicolumn{4}{|c|}{$\alpha \leq 0,05$ e $\mathrm{Pd} \geq 0,90$} \\
\hline $\mathrm{k}^{*}$ & $\mathrm{~h}^{*}$ & $\mathrm{n}$ & $\delta$ \\
\hline$\geq 0,58$ & $\geq 3$ & $\geq 1$ & $\geq 1$ \\
\hline 0,5 & $\geq 4$ & $\geq 1$ & 0,5 \\
\hline$\geq 0,34$ & 6 & $\geq 1$ & 0,5 \\
\hline
\end{tabular}

\section{REFERÊNCIAS}

COSTA, A.F.B.; EPPRECHT, E.K.; CARPINETTI, L.C.R. Controle estatístico de qualidade. São Paulo: Editora Atlas. 2004. 334 p.

KING, D.W. Statistical quality control using the SAS system. North Carolina: SAS Institute Inc., 1995. 383 p.

MONTGOMERY, D.C. Introdução ao controle estatístico da qualidade. 4. ed. Tradução por Ana Maria Lima de Farias e Vera Regina Lima de Faria e Flores. Rio de Janeiro: LTC Editora, 2004. 513 p.

MONTGOMERY, D.C.; RUNGER, G.C. Estatística aplicada e probabilidade para engenheiros. 2. ed. Tradução por Verônica Calado. Rio de Janeiro: LTC Editora. 2003. 463 p.

REYNOLDS JR., M.R.; STOUMBOS, Z.G. Control charts and the efficient allocation of sampling resources. Technometrics, v. 46, p. 200-214, 2004.

CROWDER, S.V. A simple method for studying run-length distributions of experimentially moving average charts. Technometrics, v. 29, p. 401-407, 1987.

HAWKINS, D.M. A fast accurate approximation for average run lengths of CUSUM control charts. J ournal of Quality Techonology, v. 24, p. 37-43, 1992.

WERKEMA, M.C.C. Ferramentas estatísticas básicas para 0 gerenciamento de processos. Belo Horizonte: Fundação Christiano Ottoni, UFMG, 1995. 404 p. 
